\title{
Il "ritorno" del Martello e una recente edizione del suo Teatro
}

\section{Franco Fido}

Come tanti altri scrittori, Pier Jacopo Martello si preoccupava della fama che avrebbe goduto presso i posteri. Temendo che dopo la sua morte il piú giovane Scipione Maffei, fortunato autore della Merope e nemico dei versi martelliani, si mettesse a maltrattare per iscritto le sue tragedie, Martello preparò preventivamente una risposta, Il Femia sentenziato (in cui l'aedo greco, col nome lievemente mutato per anagrammare Mafei, è condannato nel regno delle ombre a star zitto, o a esprimersi solo negli aborriti settenari doppi inventati dal rivale), e ne fece stampare un certo numero di copie, che avrebbero dovuto esser diffuse solo nel caso del temuto attacco da parte del nobile veronese, o altrimenti distrutte.

Per fortuna, anche se apparentemente la precauzione di Martello si rivelò inutile, qualche copia del Femia a stampa e parecchie manoscritte si sono salvate. Ma il bolognese non diffidava a torto delle idiosincrasie della posterità e dei capricci della fortuna. Celebre in vita, e membro rispettato di quella ideale coterie che riuniva in una complessa rete di emulazioni e amicizie i maggiori letterati e grandi Arcadi della penisola, dai venerati precursori Maggi e Lemene a Muratori e Conti, Orsi e Manfredi, Gravina e Crescimbeni, i fratelli Zeno e i coniugi Zappi, Martello fu trascurato dalle generazioni successive ("il Teatro italiano del Martello ... è deserto da un secolo e mezzo," scriverà nel 1869 Prospero Viani), ${ }^{1}$ per essere riscoperto una prima volta dai positivisti (gli studi di Saviotti, Dejob, Bertana, Galletti, Toldo ecc. sulla tragedia o sui rapporti fra la drammaturgia francese e quella italiana), ${ }^{2}$ e una seconda volta, dopo un'altra piú breve eclissi, in questi ultimi tren$t^{\prime}$ anni.

Se non all'inizio, almeno al centro del recente "ritorno a Martello" stanno le edizioni curate da Hannibal S. Noce per gli "Scrittori d'Italia" di Laterza: gli Scritti critici e satirici nel 1963, che furono accolti con interesse e favore dagli specialisti, ${ }^{3}$ e il Teatro pubblicato in tre volumi fra il 1980 e il 1982. 
Quindici tragedie, una decina tra commedie, tragicommedie, drammi satirici, piú varie favole per musica, pastorali, marittime, ecc.: si tratta di una considerevole impresa editoriale e di un'importante aggiunta al corpus dei classici laterziani, che sollecita due discorsi diversi. Uno, necessariamente sommario in sede di recensione, sull'immagine che tutti i contributi critici recenti ci permettono di ricostruire - e ora di verificare agevolmente sui testi - di un Martello praticante e teorico del teatro in un tempo e in uno spazio culturale precisi: Bologna fra la fine del Seicento e il 1727, anno della sua morte; l'Arcadia bolognese fra Roma e la Francia. L'altro sui criteri, i pregi e i limiti dell'edizione del Teatro testé pubblicata.

Se il merito che i critici della scuola storica riconoscevano piú volentieri al Martello era quello d'aver fornito al Parini, col Femia sentenziato, un confessato modello per gli sciolti e la complessa sintassi poetica del Giorno (iperbati, latinismi, ecc.: si vedano le analisi di Gnoli, Carducci e Toffanin) ${ }^{4}$ nelle pagine del Croce e in quelle del Fubini sul dialogo Il vero parigino italiano, le une e le altre del $1949,{ }^{5}$ è piuttosto il teorico della letteratura a prender rilievo, il saggista capace di conciliare l'intransigente petrarchismo di Crescimbeni con l'interesse, vivacissimo a Bologna, per il teatro francese, e al tempo stesso (in polemica coi classicisti cartesiani d'Oltralpe) con una cauta accettazione dell'eredità secentesca, per il riconoscimento che essa comportava della fantasia e dell'arte.

L'attenzione dei critici si è poi estesa al teatro e alla poesia lirica del Martello (Franco Croce, Giacinto Spagnoletti) ${ }^{6}$ comunque il ritratto piú equilibrato e ricco resta quello di Water Binni, Pier Jacopo Martello e le sue commedie "per letterati," raccolto in volume nel 1963, ma frutto di ricerche degli anni ' 50 ' che rende giustizia tanto all'acutezza del critico quanto alle virtú del commediografo: apprezzate queste sia nella direzione di un "gioco linguisticoletterario" sulle mode poetiche del tempo, che presuppone "un lettore al corrente della situazione letteraria arcadica" (Che bei pazzi), sia in quella anche piú originale dello squisito, malizioso miniaturismo con cui le passioni umane, dall'amore all'ambizione politica, sono rappresentate nella farsa per marionette o bambocciata, già cara al Goldoni, Lo starnuto d'Ercole. ${ }^{7}$

A questa fase di riscoperta del Martello è seguita in anni ancora piú vicini a noi una tendenza a lumeggiare analiticamente lo sfondo culturale della sua attività, o aspetti particolari di questa. 
Antonio Franceschetti ha giustamente insistito sulla peculiare situazione dell'Arcadia bolognese, impegnata a difendere i propri ideali letterari su due fronti, contro il Secentismo, secondo i dettami del classicismo romano e graviniano, ma anche contro la condanna della tradizione poetica italiana che nelle pagine di Boileau e del padre Bouhours risaliva dal Marino al Tasso. ${ }^{8}$

Anna Dolfi, in un'attenta rilettura degli scritti di poetica del Martello (i Sermoni, le Satire, e specialmente il Comentario pubblicato col Canzoniere nel 1710) da una parte ha proposto un parallelo fra la cultura letteraria e la cultura pittorica bolognese nel primo Settecento sotto il segno di un comune, moderato eclettismo ("Raffaello corretto dai manieristi per arrivare alla pittura del Seicento, Petrarca filtrato dai marinisti per l'approdo alla poesia della vera Arcadia, mediatrice equilibrata del bene e del male poetico"), suggerendo dall'altra di vedere nella dichiarata esclusione di ogni finalità civile dalle ricerche puramente letterarie del Martello un elemento critico dissacrante, o "ironizzazione della stessa inutile funzione della poesia." 9

Simonetta Guidi Ingegno ha ricostruito sulla base di minuziose esplorazioni d'archivio il ruolo centrale del marchese Orsi come tramite fra il teatro francese contemporaneo e i maggiori letterati di Milano e di Modena, Muratori in primo luogo, appassionato di spettacoli e - come risulta da molte lettere direttegli dall'Orsi assai piú spregiudicato e aperto al nuovo di quanto non appaia dalla sola Perfetta poesia: e su questo sfondo di "novità" teatrali passate di mano in mano e da una città all'altra, tradotte o parafrasate, recitate in collegi e in case patrizie, spicca l'apporto di Martello prima come traduttore poi come autore in proprio. ${ }^{10}$

Un utile consuntivo del lavoro precedente è infine quello di Grazio Distaso, che rilegge le liriche del bolognese alla luce del Comentario e dei Sermoni, e indica nell'idea di "novità" il progetto e l'emblema di un'operazione poetica tesa a ricuperare in Arcadia la lezione barocca. ${ }^{11}$

Se teniamo presenti gli interventi critici testé ricordati, in genere notevoli per impegno e qualità e cerchiamo di verificarne i risultati attraverso una rilettura dell'opera martelliana nei quattro volumi curati dal Noce (o cinque, contando le Lettere a L. A. Muratori da lui pubblicate nel 1955, $)^{12}$ alcune osservazioni o indicazioni per ricerche da proseguire mi sembrano imporsi: e le registro nell'ordine (o disordine) in cui mi si sono presentate. 
Colpisce innanzitutto il talento con cui Martello, sia negli scritti critici che in quelli teatrali, sapeva drammatizzare, rendere divertenti e accessibili delle questioni di poetica o di letteratura tutto sommato abbastanza astratte: e se certi espedienti usati piú di una volta, come il dialogo degli animali o delle ombre, il giudizio di Apollo in Parnaso o di Radamanto nei Campi Elisi, ecc., possono confermare la sua disposizione a sfruttare con intelligenza modelli del secolo precedente (Boccalini), originali e straordinariamente efficaci sono quasi sempre le sue parodie stilistiche, dall'inappuntabile petrarchismo di Messer Cecco e dal virtuosismo retorico e metrico del Cavalier Marino, ammirato perfino dal suo rivale (e portavoce dell'autore) Mirtilo in Che bei pazzi, ${ }^{13}$ agli spassosi esametri maccheronici del Pedagogo ("Te quoque, te Satyrum compassionatus Apollo, / Cui latiae non sunt intesa vocabula linguae, / Me macaronea voluit cecinisse Camena, / Possis ut orecchias dicenti aptare caprinas . . .") ne La rima vendicata. ${ }^{14}$

Non meno felice la soluzione, già notata dalla Dolfi, di campire i vari dibattiti sul teatro e sulla poesia sullo sfondo di paesaggi mutevoli e ariosi - le passeggiate dell'autore e dell'abate per Roma e per la campagna romana nel Vero parigino - o di un viaggio-inseguimento, come quello da Genova alla Francia sulle tracce del misterioso e dotto impostore che si fa passare per Aristotele nei dialoghi Della tragedia antica e moderna. E che non si tratti di meri espedienti strutturali risulta evidente dai rapporti allusivi che si instaurano fra i vari luoghi (Roma, Parigi, il monte Parnaso nel dipinto di Raffaello miracolosamente apertosi per accogliere l'autore nella visione del Comentario) e gli argomenti toccati via via nella discussione, cui la cornice odeporico-narrativa viene a conferire toni variati e suggestivi. Per questa arguzia e leggerezza di tocco vien fatto di pensare che se il modello "ufficiale" di Martello critico rimane Boileau, emulato nei Sermoni e nelle Satire con un misto di ammirazione e di rivalità, ${ }^{15}$ nel tono pacato e spiritoso degli scritti in prosa si potrebbe avvertire il ricordo di uno scrittore che era allora fra i pochissimi a conoscere la cultura inglese di prima mano, e che Martello era fra i pochi italiani a conoscere, ${ }^{16}$ quel Saint-Evremont che parlando appunto della tragedia aveva scritto: "il ne nous faut rien que de grand, mais d'humain: dans l'humain éviter le mediocre, dans le grand le fabuleux," e ancora: "les dieux nous manquent, et nous leur manquons. . . ."17

Già nei saggi citati di Binni, Franceschetti, Dolfi la mediazione tentata da Martello fra Seicento e Settecento, Barocco e Arcadia, Roma e Parigi era riscattata dal sospetto che si trattasse semplice- 
mente di una blanda (e, per intenderci, "qualunquistica") tendenza a salvare la capra del Marino e i cavoli del Crescimbeni. L'equilibrata posizione del bolognese si capisce anche meglio alla luce di un altro suo atteggiamento rilevato dagli stessi studiosi, e cioè la sua riluttanza a postulare un contenuto civile, politico, o in generale etico nella letteratura, cosí come questa poteva essere praticata e recepita dalla sua società e nel suo tempo, visto che "questa bell'arte non è di quelle le quali sieno al commercio dell'onesto vivere necessarie. ${ }^{18}$ Da una parte si direbbe che Martello si compiaccia a spingere quietamente fino alle sue estreme conseguenze logiche quella rarefatta interpretazione delle lettere in genere, e del petrarchismo in particolare, che era prevalsa in Italia fin dal Bembo - si ricordi il fastidio espresso nelle Prose della volgar lingua per le ambizioni totalizzanti di Dante, il quale "quanto ancora sarebbe egli miglior poeta che non è se altro che poeta parere agli uomini voluto non avesse nelle sue rime." ${ }^{19} \mathrm{Dal}-$ l'altra la cautela e lo scetticismo appaiono un segno dei tempi abbastanza diffuso, anche fuori d'Italia, fra i letterati "integrati" del primo Settecento. Si confronti quanto dice il Segretario Cliternate al Baron di Corvara nel proemio delle Satire: "Amico lettore, satira è un nome odioso. . . . In oggi la satira de' vizî morali è quasi bandita, imperocché questi con invettive piú giovevoli e ancora piú sante vengono da' sacri oratori nelle prediche loro perseguitati, ${ }^{20}$ con quel che diciassette anni prima Congreve dichiarava al pubblico nel prologo di The Way of the World: "Some plot we think he has, and some new thought, / Some humor, too, no farce but that's a fault. / Satire, he thinks, you ought not to expect: / For so reformed a town who dares correct? / To please this time has been his sole pretense; / He'll not instruct, lest it should give offense" (vv. 29-34). Infine, e soprattutto, sarà piú agevole apprezzare l'ineguagliabile nitore del Petrarca e del Casa, senza dover perciò disprezzare le rutilanti invenzioni del Marino, quando appunto si sarà capito che si potrebbe vivere anche senza letteratura.

Alla base della serenità di giudizio del Martello sta una lucidità, un senso delle proporzioni e del limite che fanno di lui per temperamento una specie di antigravina o di antibaretti, ma che rendono le sue convinzioni tanto piú ferme ed efficaci quanto piú raggiunte, per cosí dire, attraverso un malthusianismo programmatico. Ed è proprio sul terreno del teatro che troviamo la miglior conferma a questa osservazione. Nessuno fra i letterati contemporanei capí meglio di lui il ruolo giustamente dominante della musica nel melodramma ("tale che solleva gli animi da tutte le cure, e gli assorbe in una spensierata quiete che di sé contenti li 
rende. . . . Ma bisogna supporre per fondamento che in questo vago spettacolo non dee negarsi la preminenza della musica" ${ }^{21} \mathrm{e}$ la forza spettacolare, la carica di divertimento della Commedia dell'arte non solo presso un pubblico popolare ("quando cotesti artegianelli o barcaiuoli vanno al teatro per ridere, piú tosto il Dottore, il Pantalone, ed Arlecchino, e Finocchio, che la Lena, il Negromante, i Suppositi, la Cassaria, e la Scolastica vorrebbero ritrovarvi: conciossiacosaché nessuna commedia ridevole, per savia, piccante, e costumata che siesi, può alla commedia istrionica italiana resistere"), ma anche presso le persone colte, come si vede dal cordiale elogio delle maschere cui si abbandona il Martello, per concludere: "confesso ch'io lascerei l'Edipo di Sofocle, e l'Anfitrione di Plauto per una di queste favole da valenti istrioni rappresentata." 22

Ma quel che rende la commedia improvvisa cosí straordinaria - "le grazie dei dialetti," e soprattutto "gli atti, e il gesteggiar curioso, faceto, fallico e sconcio" - non si può né scrivere né stampare, talché il segreto degli istrioni è ormai fuori portata della letteratura. Non sarà dunque uno scandalizzato rifiuto dell'indigenza intellettuale e della scurrilità dell'Arte a guidare Martello nella composizione delle sue proprie opere teatrali, bensí la scoperta che in Italia i letterati avevano in sostanza perduto ogni giurisdizione sugli spettacoli in quanto occasioni di divertimento popolare, e che dunque bisognava rassegnarsi a una drammaturgia e a un raggio d'azione molto piú ristretti.

La diagnosi del Martello è in fondo piú ottimistica e tollerante di quella del suo amico Lelio, che giungerà a condannare moralisticamente quella stessa, grande tradizione attoriale da cui era uscito, e ad invocare contro le sconcezze dell'Arte una censura di stato: ${ }^{23}$ ma proprio a certe pagine del Riccoboni sulla recitazione fanno pensare le minute osservazioni del bolognese sui rispettivi meriti e vizi degli attori francesi e italiani - ulteriore prova di quanto vivo fosse in lui il senso del teatro come concreta produzione scenica. ${ }^{24}$

Davanti a un divorzio fra teatro e letteratura che Martello poteva a ragione giudicare insanabile (visto che nessuno, allora, avrebbe potuto prevedere il miracolo di Goldoni), le sue "commedie per letterati" cosí ben caratterizzate dal Binni non volevano essere una superciliosa risposta ai rozzi canovacci degli istrioni, bensí un consapevole, se pur raffinato, pis aller.

Se i letterati non possono rivaleggiare con la viscerale teatralità degli istrioni, resta loro il compito di ricuperare, classificare e criticare con garbo la tradizione teatrale colta: "l'autore ha avuto in 
idea di comprendere nel suo Teatro tutto ciò che secondo il sistema antico e moderno o è stato o è rappresentabile," leggiamo nell'autobiografia di Martello; ${ }^{25}$ e nella Dedica del suo Teatro al Senato di Bologna si afferma che questo "la ditirambica, la tragedia, la farsa, la satirica, la pastorale, la maritima, il dialogo, la rappresentazione, e tutto quello insomma che da' teatri non musicali fu rappresentato, o si rappresenta, contiene e abbraccia." 26

Questo carattere che Martello sottolinea nella sua opera, di catalogo o "teatro" enciclopedico dei sottogeneri teatrali, si ricollega a tradizioni medio e tardo cinquecentesche, e può effettivamente farcela interpretare come consuntivo tra pedantesco e nostalgico di un patrimonio ormai inerte e svalutato davanti alla realtà degli spettacoli e dei pubblici del primo Settecento.

Ma dobbiamo anche tener conto della preferenza confessata dal bolognese per un settore della sua abbondante produzione, e cioè per le tragedie. Parlando ad Antonio Conti delle reazioni suscitate in lui dal successo della Merope del Maffei egli scrive: "Potete voi credere, che alla passione mia, cioè alla passione di tale, che a questo componimento ha i migliori anni dell'età sua dedicati, piaciuto sia, che un ingegno in tante altre liberali arti e scienze esercitatissimo prorompa improvvisamente in una Tragedia, alla quale debba io quasi rendermi vinto, e per cosí dire ceder l'armi senza contesa?"27

Di questa passione le tragedie ripubblicate dal Noce offrono un documento imponente: le basi, direi, per concludere che Martello è l'autore tragico del Settecento prealfieriano di gran lunga piú interessante e degno di studio.

Un primo rilievo riguarda la natura e la funzione dei suoi martelliani, che in virtú di un attento gioco di pause e di enjambements sono molto meno ritmati e cantabili di quelli dei suoi imitatori, e rappresentano la efficace risposta a una ricerca di "locuzione Tragica, che paia prosa legata" ("ma se poi la slegarete alla prova vedrete non esser prosa, et avere anche questa sorta di locuzione il suo Poetico, quantunque assai naturale, e modesto"). ${ }^{28}$ Per capire cosa si intenda qui per "poetico naturale e modesto," si confrontino dei martelliani di Goldoni, per esempio i primi versi del Molière:

Eh via, Moliere, amico, mostratevi gioviale; un autor di commedie, un uom che ha tanto sale, che con le sue facezie fa rider tutto il mondo, co' propri amici in casa non sarà poi giocondo?

con altri presi a caso dalle tragedie martelliane: 
Ah signor, soffrirai che il piú nobil sostegno della gloria ottomana, degli anni tuoi, del regno sia prigionier? (Roselane, in La Perselide, I, 3: Teatro, II, 304)

Possan due tortorelli, de' quai sempre l'un geme quando lontano è l'altro, prima abborrirsi insieme che mai si turbi, o consorti, la vostra compagnia. . . .

(Balame, in La Rachele, I, 1: ibid, 493)

Da un lato il linguaggio della tragedia deve essere quello dei personaggi, non quello dell'autore, e quindi "il tragico non dee partirsi da uno stile che contrafaccia la forma corrente del favellare." 29 Dall'altro si tratterà sempre, appunto, di una contraffazione, o "imitazione imperfetta," ma non può essere che cosí, ed è bene che sia cosí: "l'imitazione . . . in alcune cose dee convenire, in alcune disconvenire, altrimenti non sarebbe piú imitazione del vero, ma il vero medesimo." 30

Sul vero, l'imitazione teatrale del vero presenta il vantaggio d'essere dilettevole per una dolcezza dello stile che - esclusa la pratica indiscriminata delle figure e dei tropi, plausibili nel linguaggio retorico dell'autore, non in quello "naturale" dei personaggi - non potrà venire che dal metro, ${ }^{31}$ cioè (e qui la boucle del ragionamento si chiude con perfetta logica) da versi parlabili, che "pareranno prosaici" solo "a cagione del pregiudicio in cui sono le Teste Italiane di voler per tutto ornamenti da lirico, e forme di dire o Petrarchevoli o Chiabreresche. "32

Agli stessi criteri di "imitazione imperfetta" (valido per la poesia in generale) e di "maraviglia ragionevole" (applicabile in particolare alla tragedia) si ispira la preferenza del Martello - vero e proprio partito preso strutturale - per l'ampiezza dei discorsi fatti pronunciare ai suoi personaggi: "sebbene i discorsi tragici appaiono lunghi, non lo saranno, paragonati a quel vero che si vorrebbe dalla materia, e che in grazia dell'uditorio si abbrevia, ma si abbrevia in modo che l'imitazione moderi il vero, non lo distrugga." 33

Le tragedie del bolognese presentano in azione, per cosí dire, i principi di poetica appena riassunti, e la controprova di un fatto già notato, che la forza del Martello sta nella sua capacità di riconoscere i limiti della situazione letteraria in cui si trova ad operare, e di volgerli a suo vantaggio. Stretto fra il linguaggio della passione che trionfava nel melodramma, e quello degli istinti monopolizzato dalla irresistibile gestualità degli istrioni, il teatro letterario poteva salvarsi solo sul terreno di un "mezzo realismo." Sul versante della commedia questo lasciava la strada aperta a biz- 
zarre invenzioni e stilizzazioni simboliche: la follia vedovile e il petrarchismo di Sostrata (Che bei pazzi), la pecora inconsapevolmente piú abile della volpe (A re malvagio consiglier peggiore), le lettere dell'alfabeto che si combinano a formare il personaggio del Satiro, la donna fiorentina che muore di parto nel vano tentativo di pronunciare la $c$ intervocalica senza aspirarla, e suo figlio nato morto che dall'altro mondo cerca di farsi un'idea di questo (Il piato dell' $^{\prime} H$ ). Sul versante della tragedia, al contrario, il ragionato abbandono della mimesi ad ogni costo favorisce un agio analitico, che approda a sua volta a un "realismo" non piú linguistico o gestuale, ma psicologico. Si prenda per esempio L'Edipo tiranno (1723), che se è vero quanto afferma l'autore fu la prima concepita e l'ultima composta (e dunque dovrebbe essere la migliore) delle sue tragedie. ${ }^{34} \mathrm{Da}$ una parte la lingua media, al tempo stesso nobile e colloquiale, teorizzata nei trattati Del verso tragico e Della tragedia si estende qui lungo una gamma che va dalla robusta logica di Edipo:

... io volea con un crine, qual mi levai, sconvolto mostrar dell'auree bende scinto alle morti il volto, quasi che mi paresse non so quai privilegi d'immunità dai mali dar la porpora ai regi, e dalla comun strage quai sacre ir rispettate per voler dei destini le fronti incoronate. (I, 1: Teatro, III, 570)

al "parlato" familiare di Creonte:

Cantan inni i garzoni, né in pace al Ciel l'orecchie lascian col pisipisse le suocere e le vecchie; (Il, 2: p. 592)

o agli scherzi dei soldati sui vezzi di Manto e la presunta ingenuità di Tiresia, che prende per sé i saluti rivolti invece alla figlia:

O come passa in fra 'l mirar di molti la leggiadra donzella, che dal vedere in lei girarsi i volti, sempre conosce più quant'essa è bella!

Forse ch'anche per via fra ciglia e ciglia tanto barlume accoglie che può occulto spiar che fa la figlia; poi nelle patrie soglie provvido la consiglia con quello star, con quell'andar ritroso a più infiammar le voglie onde astuta s'acquisti e dote e sposo. (III, 5: pp. 606-07) 
Dall'altra il comportamento e le motivazioni dei personaggi sono frugati nelle loro zone piú oscure, come quando Jocasta osserva che se i tebani soffrono per volontà degli dei le conseguenze di chissà quale colpa collettiva, ciò non dovrebbe impedire a lei e ad Edipo di godersi insieme un po' di felicità; o quando la stessa regina ricorda lo strano comportamento del nuovo marito durante la prima notte di nozze, dopo che il giovane aveva riconosciuto nel simulacro di Laio l'uomo da lui ucciso in Focide, e le sue proprie reazioni:

E questo era, o infelice, che con fronte abbattuta

ti diportasti allora da un uom d'età canuta,

e ch'io correr sentimmi piú foco a questa faccia

d'allor che Laio accolsi fanciulla in fra le braccia.

Era il nostro accostarci qual di chi ruba, e pave,

e gli atti in sé soavi nulla avean di soave. (I, 2: p. 579)

A esiti di questo tipo, non privi di audacia, mi riferivo poco fa quando parlavo di "realismo psicologico": una dimensione piú "da romanzo" che da teatro, se si vuole, che si ritrova nell'intervento indiretto dell'autore quando fa dire a Edipo, prima da Jocasta, poi da Tiresia, quello che noi spettatori-lettori dobbiamo pensare di lui:

Mentre, o signor, parlando m'apri piú e piú la mente,

né piú sí reo ti scopro, ma né piú sí innocente (I, 2: p. 583)

e non è forse rea, qual sembra altrui, tua mente;

ma non quanto a te sembra, tanto è forse innocente.

(V. sc. ult.: p. 634)

Anche nelle tragedie dunque (come mi pare risulti dalle ultime citazioni) si manifesta la tendenza a vedere una mistura di male e di bene là dove un osservatore manicheo non vedrebbe che l'uno o l'altro; e a tale tendenza si può ricollegare il gusto del Martello per certi testi che sembrano come sospesi fra commedia seria e tragedia, quali l'Anfitrione di Plauto o il Don Sanche di Corneille. ${ }^{35}$ In questo senso, anzi, molte delle tragedie di Martello, e delle piú notevoli, come I Taimingi o L'Elena casta, ammetterebbero l'etichetta di tragicommedia o di commedia eroica, che egli riserva invece a due sole, rispettivamente La Morte di Nerone e Il Davide in Corte. L'interesse di quest'ultima, in particolare, oltre che nei confronti cui si presta con testi di altri autori (dal Du Ryer - per Saul, se non per David - all'Alfieri), sta nella compiutezza con la quale 
sfrutta tutti i toni e gli ingredienti del teatro martelliano: dalle invenzioni "lucianesche" al confine del macabro (l'ombra di Golía che contempla infastidita il proprio teschio pendente dalle mura di Gerusalemme), agli spunti comici o semicomici, come quello di Davide inseguito da un codazzo di canore ammiratrici ("Si sa quel che in Davitte stuol di fanciulle apprezza: / Fingon lodi al valore, ma danle alla bellezza," osserva Saul: II, 1: Teatro, II, 174), o impaurito dalle avances della cognatina Merobe ("Siedi, non son già spine que' morbidi origlieri, / Che sfuggevole, e in atto piú che di star, di gire, / Qual gru col piè sospeso, temer sembri al mio dire": II, 4: p. 182).

La costante intertestuale va di nuovo ravvisata nella mediocritas dei personaggi, anche quando dovrebbero essere del tutto buoni o del tutto cattivi. Cosí Saul, il tiranno che nel corso della pièce si rivelerà sempre piú odioso, è capace parlando con Abner di riconoscere la lealtà di Davide, e di veder chiaro in se stesso:

... avverti: io non vorrei che traditor paresse, ma che lo fosse, e il fosse a gli occhi insin di Jesse, perch'io bisogno allora terrei di men soccorso dal mio interno livore contro del mio rimorso. (III, 4:Teatro, II, 201)

Mentre il casto e timorato Davide non è insensibile alle grazie naturali e al sex appeal di una pastorella,

che in suo farsetto avvolta di monda agile tela va piú leggiadra e sciolta, tal, che il lineamento del torsicciuol, de' fianchi

traspar nulla alterato dai tesi lini e bianchi. (III, 2: p. 194)

In Davide (che non per nulla è poeta) l'opposizione Corte-selve, vita principesca-vita pastorale è presentata in termini prettamente arcadici. Ma ecco immediatamente, a ridimensionare e demistificare i facili sogni idillici del marito, l'interessato buon senso di Micol, che non è affatto disposta, per seguire lui esule nei boschi, a rinunciare alle comodità godute fin dalla nascita:

Fingimi un po', consorte, di questi manti in vece rafazzonata in gonne del color della pece, colle man careggiarti fresche dell'aver munte le mandre, e de' conditi cibi ancor lorde e unte; e sospirarti in viso, come la tua Micolle, ma in sospiri olezzanti sol d'agli e di cipolle; (III, 1: p. 192) 
né migliori dei giorni sarebbero per lei le notti, accanto a uno sposo abbrutito dalle fatiche:

... né a povertate, né al soffrir carmi avvezza

mi vedrò disadorna mancar la mia bellezza, onde ognor men piacendo a chi suol colta amarmi, piú sempre a lui posposta vedrommi a que' suoi carmi; e coi membri dal nuovo suo faticar dirotti meno a me saporose dormir vorrà le notti, poich'altro è l'agitarsi dietro le belve in caccia, altro è il seder cantando coll'arpa in fra le braccia. (IV, 4: p. 220)

I personaggi del Martello "si vedono" e si accettano come sono, senza farsi illusioni su se stessi. La loro maggior risorsa sta appunto nella lucidità con cui assumono le proprie debolezze: in questo senso il conflitto fra Davide e Micol è una metafora teatrale della disposizione martelliana a prendere sempre in considerazione i possibili eccessi (di un personaggio, buono o cattivo; di una poetica, castigata o ridondante), senza che essi lo scoraggino dal cercarne le ragioni, dall'apprezzare i meriti di quel personaggio, di quella poetica.

Se il teatro come spettacolo era, per cosí dire, sfuggito alla letteratura, Martello pensò di ricuperarne almeno un pezzetto, offrendo sulle scene quello che né i comici dell'Arte né i virtuosi dell'opera in musica potevano dare: una ragionevole analisi da molteplici punti di vista e, come avrebbe detto Ennio Flaiano, "sul piano corretto dell'alta conversazione," di varie situazioni e di vari caratteri: quasi un surrogato di quel romanzo che in Italia restava tabú per i letterati che si rispettino.

Tuttavia sarebbe errato concludere che le tragedie del Martello erano destinate solo alla lettura, e non alla rappresentazione. Piuttosto (come la pratica teatrale di questi ultimi decenni sembra suggerire a proposito di Manzoni, contro lo stesso autore) si potrebbe dire che il suo teatro era la sceneggiatura, spesso efficace, di testi concepiti a partire da un codice letterario: sia, come si è visto, nel genere serio sia in quello comico, dove la complicità scherzosa coi destinatari, l'allusione a opere e ruoli consacrati, potrà essere di per sé fonte di divertimento.

Senza voler istituire assurdi confronti qualitativi, o proporre non meno strampalate derivazioni, ma come illustrazione di un possibile futuro per il "teatro parallelo" intuito da Martello, si pensi a certe linee ipotetiche e divergenti: dalla sua Alceste all'Ermengarda di Manzoni, dalle sue commedie aristofanesche alla Finestrina di Alfieri, dalla sua Elena casta allo scintillante libretto di 
Meilhac e Halévy per la Belle Helène di Offenbach. Del resto, che il bolognese possedesse fra tanti altri doni una vocazione di futurologo è ben evidente nel suo straordinario trattato fantascientifico Del volo.

Venendo al lavoro ecdotico di Hannibal Noce, alcune delle critiche che gli si potrebbero rivolgere preliminarmente e in astratto non sono veramente giustificate. Come nel volume del 1963 Scritti critici e satirici poteva spiacere la mancata inclusione dell'interessante e breve autobiografia, ${ }^{36}$ cosí nelle Appendici ai tre del Teatro si può rimpiangere l'assenza di qualche pezzo minore di un certo rilievo drammaturgico, come l'Apologia premessa alla Parte prima del Seguito del teatro italiano ... (cioè al vol. IV delle Opere: 1723), o la Lettera al Conti già ricordata. Il curatore si è inoltre limitato, per ogni opera inclusa nella sua edizione, a fornire con molta cura la tradizione testuale e la fortuna teatrale, rinunciando invece a darne un commento storico-esegetico, che sarebbe stato particolarmente utile nel caso delle dediche e dei proemi, in cui Martello cita e discute altri autori. La rete di letture, citazioni magari a memoria, rimandi eruditi è cosí fitta nella cultura settecentesca, che ogni nuova edizione di opere di quel secolo diventa un'occasione di chiose, verifiche, repertori; in altri volumi della grande collana laterziana tale occasione era stata sfruttata meglio, per esempio nei Saggi di Algarotti curati da Giovanni Da Pozzo nel 1963. Ma, ripeto, queste osservazioni riguardano piú la direzione della collana, la maggiore o minore coerenza di criteri cui essa si informa, che non la responsabilità dei singoli curatori.

Diverso è il discorso da fare sul testo. Primo merito del Noce è quello di aver rintracciato e pubblicato (per la prima volta insieme in un'edizione di opere del Martello) secondo le stampe originali varie sue composizioni teatrali minori, scritte e rappresentate per lo piú negli ultimi anni del Seicento: le "favole per musica" Il Perseo, La Tisbe, Apollo Geloso, Gli Amici; l'oratorio S. Caterina Vigri, la pastorale La Ninfa costante, la favola piscatoria Il Reno pensile.

Per le opere teatrali già raccolte nelle maggiori edizioni settecentesche del Martello, il logico criterio seguito dall'editore è stato, quando si trattava di scegliere fra varie stampe, di privilegiare l'ultima corretta dall'autore (che sarà nella maggior parte dei casi quella romana del 1715 o quella bolognese del 1723), ${ }^{37}$ dando eventualmente in nota le varianti dell'edizione principe (quella romana del 1709), o di qualche stampa isolata (come quella veronese 
della Rachele, 1719). Cosí per le prime dieci tragedie (La Perselide, Il Procolo, L'Ifigenia in Tauris, La Rachele, L'Alceste, Il M. Tullio Cicerone, L'Edipo Coloneo, Il Sisara, Il Quinto Fabio, I Taimingi) Noce segue l'edizione romana del 1715 , che fu poi riprodotta senza mutamenti dal Dalla Volpe in quella bolognese del 1735. Dalla stessa edizione romana è preso il testo della favola marittima $L^{\prime} A d r i a$, del dramma sacro Il Gesú Perduto, della tragicommedia La Morte di Nerone.

Per le cinque tragedie non incluse nell'edizione romana del 1715 (Il Catone, L'Elena Casta, Il Perseo in Samotracia, La Morte, L'Edipo Tiranno) Noce riproduce il testo del Seguito del teatro italiano di $P$. J. Martello, Parte prima e Parte seconda, cioè dei volumi IV e V delle Opere. Da questi stessi volumi IV e V dell'edizione bolognese è preso il testo delle commedie e dei drammi satirici (Che bei pazzi, A re malvagio ... Lo Starnuto di Ercole, Il Piato dell'H, La Rima vendicata), del ditirambo $L^{\prime}$ Arianna, della commedia eroica Il $\mathrm{Da}$ vide in Corte.

Per Il Femia sentenziato infine Noce è ricorso, come già Prospero Viani nel 1869, all'esemplare dell'edizione principe (Cagliari, Fr. Anselmo: ma Milano, Stamperia Palatina, 1724) postillato dallo stesso Martello e conservato alla Biblioteca Universitaria di Bologna.

Con due eccezioni (la lettera Della ritirata del Femia di cui dirò appresso, e il Muzio Scevola tradotto dal francese del Du Ryer, riprodotto secondo un manoscritto dell'Universitaria di Bologna piuttosto che dall'unica stampa) tutti i testi pubblicati dal Noce erano reperibili solo in attendibili edizioni dell'ultimo Seicento o del Settecento: e questo limitava e insieme semplificava il compito del curatore. Tanto piú stupisce l'abbondanza di errori - difficile dire, spesso, se di stampa (cioè imputabili al correttore di bozze) o di trascrizione (e quindi dovuti all'editore).

A titolo indicativo, ho collazionato con l'originale le opere seguenti: Che bei pazzi (ultimi due atti), A Re malvagio consiglier peggiore, Lo Starnuto di Ercole, Il Femia sentenziato, ${ }^{38}$ Il Piato dell'H, La Rima vendicata, Il Davide in Corte, L'Edipo Tiranno, che rappresentano, come numero di pagine, il $20 \%$ di tutta l'edizione in tre volumi; e do qui di seguito gli errori rilevati, facendo seguire la lezione del Noce da quella corretta.

Vol. I: p. 305, verso 554: cerbero per cerebro; p. 306, v. 594: è per $e$; p. 339 , riga 13: ronsare per ronfare; p. 378 , r. 13: trovanno per trovammo, r. 29: algebriaco per algebraico; p. 411, v. 145: e poco per è poco; p. 414, v. 416: saltellavi [cosí anche nell'originale, ma chiaramente] per saltellava; p. 417, v. 141: mari per nari; p. 521, r. 11: rifuggono. I vizziosi per rifuggono, $i$ vizziosi; p. 529, v. 7: $t i$ per di, v. 
17: sceuri [anche nell'orig., ma chiaramente] per scevri; p. 534. v. 159: apposta per opposta; p. 539, v. 313: quanto per quando; p. 541, v. 366: Alighiero per Alighierio (in rima!); p. 559, v. 76: direi la [orig. direile] per direila; p. 563, v. 201: dissimile per dissimule, v. 209: rincunciar per rinunciar; p. 568, v. 383: se per sé; p. 574, vv. 81-84: il punto fermo alla fine del v. 82 e la virgola alla fine del v. 84 vanno scambiati di posto; p. 584, v. 438: tural per turan; p. 606, v. 432: chiusa per schiusa; p. 617, v. 307: se si per se mi; p. 618, v. 348: a noi giova per a noi non giova; p. 622, v. 469: È fama per E fama; p. 629, v. 109: se non e. . . cosa e per se non è . . cosa è; p. 637, v. 6: vorrei, se per vorrei, che se; p. 638, v. 31: è per e; p. 639, punto e virgola invece di virgola alla fine del v. 86; p. 643, v. 203: sé stessa per sé tessa (voce del v. "tessere"); p. 647, v. 102: gradía o guerra per gradía che, o guerra, v. 109: punto e virgola invece di punto alla fine del v.

Vol. II: p. 168, v. 135: dir per di; p. 176, v. 77: dilettando per dilatando; p. 178, v. 130: prendi ancora per prendi tu ancora; p. 186, v. 389: a un brun per a brun, v. 403: sorditi per sordidi; p. 187, v. 422: incensati per incènsasi; p. 189, v. 1: lagrimante per lagrimate; p. 191, v. 72: sorgean per sorgea; p. 198, v. 291: questa reggia per questa sua reggia; p. 203, v. 464: corrier per corsier; p. 204, v. 494: e del grano per e il grano; p. 210, v. 106: interpreti per interpetri; p. 215, v. 274: $d i$ per sí; p. 222, v. 494: è soffrir per è a soffrir, v. 498: e a te per $e$ da te; p. 227, v. 105: è per e; p. 230, v. 200: la segua per lo segua; p. 232, v. 270: nemica per nemico; p. 233, v. 313: torose membra per torose tue membra; p. 234, v. 337: son grato per so grato; p. 238, v. 476: proveggio per preveggio; p. 239, v. 498: $a^{\prime}$ [anche nell'originale, ma chiaramente] per $a, v$. 517: te per $t i$.

Vol III: p. 570, v. 35: dell'altra per dall'altra; p. 573, v. 150: l'intenderne per d'intenderne; p. 575, v. 217: mail per mai; p. 576, v. 257: e per è; p. 581, v. 439: ha per hai; p. 587, v. 48: sin fan per si fan; p. 589, v. 107: balià per balía; p. 594, v. 267: defonto. per defonto,; p. 597, v. 362: Dire per Dive; p. 603, v. 110: Corinto per Corintio; p. 605, v. 176: lei sempre per lei per sempre; p. 606, v. 221: solti per folti; p. 610 , v. 26: malatti per mal atti; p. 616, v. 227: ché per che; p. 619, v. 323: e per è; p. 621, v. 380: fu per fur; p. 626, v. 64: più orrendo per più d'orrendo; p. 628, v. 139: avviene per avvien; p. 629, v. 185: rificandosi per rificcandosi.

Piú di 70 errori (e la loro banalità e distribuzione regolare) in un numero di pagine - 433 - che come dicevo rappresenta il $20 \%$ di quelle complessive - circa 2130 - inducono a ipotizzare nei tre volumi qualche centinaio di errori: una cifra che mi sembra molto 
difficile giustificare o scusare in una edizione del prestigio (e del prezzo) di questa laterziana. ${ }^{39}$

Il problema che si poneva per la pubblicazione della lettera apologetica sul Femia era ancora differente. Già il Viani, nella sua edizione del 1869 (che è l'unica a stampa completa prima di questa) si era servito "de' codicetti conservati nelle biblioteche della R. Università di Bologna, dell'Archiginnasio, e dei Principi Hercolani, "40 ma senza spiegare i criteri di scelta fra lezioni diverse nei vari manoscritti. Il Noce dichiara di averne consultati nove: 4 dell'Universitaria di Bologna, 3 della Biblioteca Comunale dell'Archiginnasio, e due rispettivamente della Comunale di Verona e della Nazionale di Firenze, anche se poi nell'apparato di varianti non tiene conto (per ragioni che possono essere eccellenti, ma non sono spiegate) di due dei mss. dell'Archiginnasio. "Nello stabilire il presente testo" - scrive il Noce - "pur tenendo nella dovuta considerazione l'edizione Viani, abbiamo introdotto lezioni diverse legittimate dalla maggioranza dei manoscritti collazionati con questa." 41 Ora, a me sembra che, avendo lavorato il Viani con gli stessi codici usati dal Noce (anzi qualcuno in meno, come il veronese e il fiorentino), per stabilire in quale considerazione si dovesse tenere la sua edizione era necessario ricostruire e discutere i suoi criteri ecdotici: se poi questi fossero risultati vaghi o inconsistenti, la stampa del 1869 andava rimossa dalla posizione privilegiata che invece il Noce sembra assegnarle nel momento stesso in cui se ne discosta (". . . . manoscritti collazionati con questa"). Ma per criticare in modo costruttivo l'edizione di Viani, e opporre alle lezioni da lui scelte altre migliori, bisognava prima stabilire, se non proprio uno stemma, almeno un raggruppamento gerarchico di attendibilità fra i vari testimoni ( 9 o 7 che siano): e francamente il criterio della mera "maggioranza" seguito dal Noce mi pare di per sé piú sicuro nell'ambito della democrazia parlamentare che in quello della filologia testuale.

Se l'impostazione del problema lascia perplessi, è ovvio che le sue conseguenze testuali si potrebbero valutare solo attraverso un confronto coi mss. bolognesi. Anche senza ricorrere a tale confronto, si deve constatare a malincuore che Della Ritirata del Femia è pubblicata con la stessa sciatteria già rilevata nei componimenti teatrali. Ecco alcune sviste: Vol. I, p. 681, v. 3 della citazione dal Guarini: Piangero per Piangerò; p. 694, r. 27: preparasi per preparisi; p. 695, r. 8: pro per pro'; p. 696, r. 28: renduto, per renduto.; p. 697, r. 32: volevano per volevamo; p. 698, r. 32: persaudergli per persuadergli; p. 699, r. 2 della citaz. da Diogene Laerzio: salvò per salvo; p. 700, prima r.: Non che per No che. 
Per chiudere il discorso sulla Ritirata del Femia su una nota un po' meno pedantesca, vien fatto di chiedersi chi fossero i "tre amici" ai quali essa è destinata, e a quale dei tre in particolare si rivolga il Martello ("A tre amici in Italia io voglio rendere conto della mia ritirata del Femia, e voi ne siete uno"). ${ }^{42}$ Il fatto che un esperto di cose martelliane come Noce non abbia azzardato alcuna ipotesi dovrebbe indurre alla prudenza. Si potrebbe comunque arrischiare una congettura sulla base di una lettera del 16 febbraio 1724 del Gherardi al Muratori, in cui si legge tra l'altro: "Attenderò la lettera scritta dal sig. Martelli al nostro buon filosofo sig. Grassetti," e nel P.S.: "Accuso l'altra sua lettera de' 3 del corrente, con la quale ho letta la scritta del sig. Martelli al nostro sig. Grassetti. E poicché l'autor suddetto non si è saputo dispensare da dar certe pennellate che han del satirico, non è comunicabile essa a tutti, e per conseguenza da leggersi con gran riserva. Nella dotta conversazione a cui soglio tutte le sere che posso trasferirmi, ivi la mostrai, e gli amici del sig. Muratori n'ebbero piacere nell'udirla, rimanendo loro solamente il desiderio che la penna dello scrittore non avesse toccato individualmente e con ispecificazione certe particolari persone che, a dí nostri, per poco che sentansi punte, rivolgonsi a mordere senza discrezione." 43 I "tre amici" potrebbero essere dunque Muratori, Gherardi e Grassetti, e quest'ultimo il destinatario della Ritirata. Il nome dell'abate Giannantonio Grassetti torna spesso nelle lettere di Martello a Muratori, fra le altre una dell'ottobre del $1725 .^{44}$

Le mende che per dovere di recensore ho dovuto sottolineare nell'edizione del Teatro di Martello curata dal Noce non tolgono ai tre volumi il merito di rendere accessibile un corpus di opere drammatiche di cui solo da poco si è cominciato a capire l'interesse, col corredo delle splendide antiporte riprodotte dall'edizione bolognese, e con un largo, sicuro inquadramento storico sulla genesi, le stampe, le rappresentazioni di ogni testo. Per le ragioni che ho detto, tuttavia, i recenti volumi laterziani non valgono veramente a sostituire la grande edizione settecentesca appena ricordata, che resta indispensabile.

\section{Brown University}

\section{NOTE}

1 Cfr. Il Femia sentenziato di Pierjacopo Martello con Postille e lettera apologetica inedite e la vita scritta da lui stesso: "Scelta di curiosità letterarie inedite o rare," disp. 100 (Bologna: Romagnoli, 1869), p. xv. 
2 A. Saviotti, "L'imitazione francese nel teatro tragico di P. J. Martello," estr. dalla Battaglia Bizantina (Bologna, 1887); per tutti gli altri cfr. Simonetta Ingegno Guidi, "Per la storia del teatro francese in Italia: L. A. Muratori, G. G. Orsi e P. J. Martello," La Rassegna della letteratura italiana, a. 78 S. VII (1974), 64-94: la nota 1 a p. 64. Per i contributi piú recenti sugli stessi argomenti si vedano l'ottima bibliografia ragionata di Guido Nicastro, in Metastasio e il teatro del primo Settecento: "LIL" 33 (Bari: Laterza, 1973), pp. 55 sgg., e la voce Martello di Gilberto Pizzamiglio nel Dizionario critico della letteratura italiana (Torino: UTET, 1974), II, 542-45.

3 Per esempio nella scheda di Walter Binni in La Rassegna della l. ital., a. 67 S. VII (1963), 360, e nella recensione di Antonio Franceschetti in Lettere italiane, XVI (1964), 221-23.

4 Cfr. Domenico Gnoli, Studi letterari (Bologna: Zanichelli, 1883), pp. 285 sgg. e spec. 332 sgg.; Carducci, "Storia del Giorno" ne Il Parini maggiore (Bologna: Zanichelli, 1907), pp. 153-65; Giuseppe Toffanin, L'Arcadia (Bologna: Zanichelli, 1946), pp. 125-43, poi ripubbl. in Storia dell'Umanesimo (ibid., 1964), IV.

5 Croce, "I versi di T. Zami e le prose di P. J. Martello," in La Letteratura italiana del Settecento (Bari: Laterza, 1949), pp. 76-92; Mario Fubini, "Arcadia e Illuminismo" (del 1949), in Dal Muratori al Baretti (Bari: Laterza, 1954), pp. 307-10.

6 Franco Croce, "P. J. Martello," La Rassegna della l. ital., a. 57 S. VII (1953). 137-47; Giacinto Spagnoletti, "E' il fanciul che qui ha chiuse le pupille . . . (Sul Canzoniere del Martello)," Paragone, n. 34 (ott. 1952), 14-23.

7 Walter Binni. "P. J. Martello ... , " in L'Arcadia e il Metastasio (Firenze: La Nuova Italia, 1963), pp. 152-68.

8 A. Franceschetti, "L'Arcadia di Roma e di Bologna nel pensiero di Benedetto Croce," in Atti del VII Convegno dell'Assoc. Intern. per gli Studi di Lingua e Letteratura Italiana (Bari), pp. 249-67.

9 A. Dolfi, "Cultura e ideologia nella poetica di P. J. Martello," Studi Urbinati, 47 (1973), 382-432.

10 "Per la storia del teatro francese . . .": cfr. nota 2 qui sopra.

11 G. Distaso, "Fra Barocco e Arcadia: poesia ed esperienza critica di P. J. Martello," Annali della Scuola Normale Superiore di Pisa, Cl. di Lettere e Filosofia, S. III, vol VI (1976), 505-27.

12 Lettere di P. J. Martello a L. A. Muratori: "Centro di Studi Muratoriani. Corrispondenti di L. A. Muratori, N. 1," a cura di Hannibal S. Noce (Modena: Aedes Muratoriana, 1955).

13 Cfr. atto IV, sc. 5, Mirtilo: "Tre sdruccioli per verso? In ver che supera / Le tue terzine, o Serafin dell'Aquila": Teatro, I, 305.

14 A. II, sc. 1: ibid., p. 557.

15 II "satirico e vanamente mordace" Boileau è ricordato nel Vero Parigino italiano (Scritti critici e satirici, p. 382), e con piú simpatia nella Dedica del Piato dell'H al conte Isolani, a proposito della satira che sa mordere discretamente: "Monsieur Boyeleau ne ha dato un perfetto esempio nel suo idioma, perseguitando colle sue innocenti e pungentissime satire la falsa letteratura, di modo che i medesimi, in derisione de quali egli ha scritto, han potuto leggerle senza disdegno" (Teatro, I, 525).

16 Il Fubini (Dal Muratori al Baretti, pp. 178-79) osserva che Sur les poëmes des anciens di Saint-Evremond era certamente noto al Muratori, e probabilmente al Becelli. Martello ricorda il francese ripetutamente e con rispetto nel trattato Della tragedia antica e moderna (pubbl. da Antonio Conti nel 1714): Scritti critici, pp. 273, 299, 303.

17 Oeuvres meslées (Paris: Compagnie des Libraires, 1692-97), III, pp. 76 e 72.

18 Sermoni della poetica, Proemio: Scritti critici, p. 4.

19 Cfr. Bembo, Prose e rime a cura di Carlo Dionisotti (Torino: UTET, 1960), p. 178.

20 Scritti critici, p. 73.

21 Della tragedia . . . , V: Scritti critici, p. 275. 
22 Dedica di Che bei pazzi a G. B. Recanati: Teatro, I, 235-36. L'importanza di questa pagina era stata rilevata dal Croce, Poesia popolare e poesia d'arte (Bari: Laterza, 1933), pp. 508-09, e dal Binni, L'Arcadia e il Metastasio cit., p. 152.

23 Del Riccoboni si veda in particolare De la réformation du théâtre (1743, poi Paris: Debure \& Lebreton, 1767), e su questa Salvatore Cappelletti. "Dalla commedia dell'Arte alla commedia borghese: Luigi Riccoboni e la sua riforma del teatro," Forum Italicum, 14 (1980), 175-94: 177.

24 Cfr. i vari scritti del Riccoboni sull'argomento: Dell'arte rappresentativa (London: Riva, 1728); le Pensées sur la déclamation che accompagnano le Réflexions historiques et critiques sur les différents théâtres d'Europe (Paris: Guérin, 1738), e l'inedito Discorso della commedia all'improvviso pubbl. da Irene Mamczarz con alcuni scenari (Milano: Il Polifilo, 1973). Del Martello si veda Della tragedia, Sessione sesta: Scritti critici, pp. 303-16. Per trovare un'analoga consapevolezza, da parte di rappresentanti della letteratura "alta" della natura spettacolare e, si direbbe oggi, "performativa" del teatro, bisogna ancora una volta cercarla fuori d'Italia, per esempio in Richard Steele: "it must be remembered a play is to be seen, and is made to be represented with the advantage of action, nor can appear but with half the spirit without it" (Prefazione di The Conscious Lovers: 1722). Ma a Londra c'erano degli spettatori teatralmente educati, e ben due "teatri stabili."

25 Cfr. Il Femia sentenziato, ed. Viani cit., p. 20.

26 Teatro, I, 664-65; cfr. anche il Proemio dell'Arianna: Teatro, II, 89.

27 ll Cesare, tragedia del sig. ab. Antonio Conti, nobile veneto, Con alcune cose concernenti l'opera medesima (Faenza: Archi, 1726); la Lettera del Martello è alle pp. 35-41: cfr. 38-39.

28 Lettere a L. A. Muratori, ed. cit., p. 49.

29 Del verso tragico: Scritti critici, p. 179.

30 Della tragedia: Scritti critici, p. 256. In queste stesse pagine Martello insiste sul "patto teatrale" fra attori e spettatori, cioè, come diremmo oggi, sulla convenuta suspension of disbelief da parte di questi ultimi, in termini che anticipano acutamente gli argomenti addotti piú tardi da Johnson, Baretti e Manzoni contro le unità di tempo e di luogo nella tragedia.

31 Della tragedia: Scritti critici, p. 301.

32 Lettere a L. A. Muratori, p. 48.

33 Della tragedia: Scritti critici, p. 306.

34 Dedica dell'Edipo alla marchesa Bentivoglio Albergati: Teatro, III, 560.

35 Per la "locuzione candida, semplice e popolare" dell'Anfitrione cfr. Del verso tragico: Scritti critici, p. 180; e anche il Proemio de La Morte di Nerone: Teatro, II, 243-44. Per la "tragedia a lieto fine" di Corneille, la Dedica alla Maratti Zappi del Davide in Corte: Teatro, II, 155.

36 Scritta come quella del Vico in risposta alla famosa Proposta ai Letterati d'Italia di Giovannartico di Porcia, e pubblicata poi dal Calogierà nella Raccolta di opuscoli scientifici e filosofici, t. II, 1729, quindi dal Viani nell'ed. cit. del Femia.

37 Per una chiara idea della successione cronologica e del contenuto delle varie edizioni - le due romane del Teatro pubblicate nel 1709 da Fr. Gonzaga; il Teatro italiano del 1715 presso lo stesso libraio, in due volumi; le Opere edite a Bologna fra il 1723 e il 1735 da Lelio Dalla Volpe in sette - si veda la Nota bibliografica del Noce agli Scritti critici, spec. pp. 502-07

38 Per il Femia, in mancanza della copia dell'Universitaria di Bologna con postille autografe, ho usato l'ed. del Viani e quella aggiunta al II vol. della Raccolta di Tragedie scritte nel secolo XVIII (Milano: Soc. tipogr. dei Classici Italiani, 1825), pp. 331-97.

39 Anche nelle Note in fondo ai voll. si riscontra la stessa negligenza che vizia l'edizione dei testi. Si veda per es. Vol. I: p. 764, riga 4: $e$ per e; p. 770 , r. 10 della lettera citata: $m i$ per vi; p. 776, r. 13:000-000 per 673-75; p. 779, r. 10: 679 per 678, r. 19: 680 per 681. Vol. III: p. 772, ultima riga: ", p. 641 per vol. III, p. 641.

40 Il Femia, ed. Viani, p. xi.

41 Teatro, I, 777. 
42 Ibid., p. 667.

43 Ediz. Nazionale del Carteggio di L. A. Muratori, Vol 20: Carteggio con Pietro E. Gherardi, a cura di Guido Pugliese (Firenze: Olschki, 1982), pp. 40-41. È vero che da vari elementi nella Ritirata si capisce che vario tempo deve essere già trascorso dalla stampa del Femia: ma è possibile che, come la città e lo stampatore, anche l'anno di questa sia falso, e che il libretto vada antidatato al 1723 . È vero altresí che vi si parla del Giulio Cesare del Conti, e della lettera a lui diretta dal Martello, come di scritti di imminente pubblicazione (uscirono nel 1726: cfr. qui sopra, nota 27); questo potrebbe essere un ostacolo piú serio alla mia congettura.

44 Cfr. Lettere a L. A. Muratori, ed. cit., p. 68. 